\title{
Heat transfer analysis for FGMs using SPH-CSPM
}

\author{
Guiming Rong ${ }^{1, a}$ and Hiroyuki Kisu ${ }^{2, b}$ \\ ${ }^{1}$ Graduate School of Science and Technology, Nagasaki University, 1-14 Bunkyo-machi, \\ Nagasaki 852-8521, Japan \\ 2 Department of Mechanical Systems Engineering, Nagasaki University \\ ${ }^{a}$ rong@nagasaki-u.ac.jp, ${ }^{b}$ kisu@nagasaki-u.ac.jp
}

Keywords: Heat transfer, Functional graded materials (FGMs), Smoothed particle hydrodynamics $(\mathrm{SPH})$, Corrective smoothed particle method (CSPM)

\begin{abstract}
The solution of heat transfer problems for functional graded materials (FGMs) by smoothed particle hydrodynamics, in which the thermal conductivity is a function of the spatial coordinates and the temperature, is discussed for both steady and non-steady problems under various boundary conditions. The boundary is treated using the corrective smoothed particle method to heighten the accuracy. Several calculations are performed to test the validity of the formulation. As an example of practical application, the problem of FGM cylindrical plates subjected to thermal shock is calculated, in which the thermal conductivity is temperature dependent and the heat transfer coefficient is varied in radial direction.
\end{abstract}

\section{Introduction}

Advanced materials known as functionally graded materials (FGMs) are now widely used in various applications, especially for severe thermal loads. Hence, many studies have been conducted regarding the properties of FGMs under severe thermal load. The distribution of the properties can be classified as discrete and continuous; one example of the latter is the conductivity variation with respect to the spatial coordinates. Ochiai solved the steady problem using the triple-reciprocity boundary element method [1]. Sakurai made Moving Particle Semi-implicit Method (MPS) formulations for both steady and non-steady problems [2]. Sladek et al. [3] and Nakonieczny et al. [4] solved this problem by the meshless local boundary integral equation method and meshfree finite element method (FEM), respectively. These approaches are for two-dimensional problems, and are coordinate dependent. However, the change in the mechanical and conductive properties of most materials with temperature when the temperature is high cannot be ignored. Kamran et al. used an integrated micromechanical-structural framework to analyze the coupled heat conduction and deformations of FGMs having temperature and stress dependent viscoelastic constituents [5]. Furthermore, Sadowski et al. evaluated the heat transfer coefficient in FGM cylindrical plates subjected to thermal shock, and compared the experiment results to those from finite difference numerical code and from FEM using ABAQUS code [6,7].

Smoothed particle hydrodynamics (SPH) is a powerful tool for the analysis of fluids and large deformation problems. It is also available for non-linear problems, because it does not require a grid and is concise in the formulation. The approach to heat conduction analysis including temperature dependent conductivity problems has been studied by Cleary et al. [8] using SPH with isothermal boundaries. In the present study, we verify the adaptability of particle methods to non-linear problems using a SPH- corrective smoothed particle method (CSPM) formulation, by which both temperature dependent and coordinate dependent conductivity problems can be treated under various boundary conditions. Steady and non-steady problems are calculated and the results are compared to theoretical and experimental results.

\section{Formulation of SPH for Heat Transfer Problems}

The governing differential equation for the transient heat transfer problem when there is no heat source in the domain is given as:

$$
\rho c \frac{\partial T}{\partial t}=\nabla(\kappa \nabla T)
$$


where $T$ is the temperature, $t$ is the time, $\rho$ is the density, $c$ is the specific heat capacity, and $\kappa$ is the conductivity. The boundary conditions can be written as

$$
T=T_{1}\left(\text { on } S_{1}\right) \quad-\kappa \frac{\partial T}{\partial n}=q-\Psi\left(T_{s}-T\right),\left(\text { on } S_{2}\right) \quad S_{1} \cup S_{2}=S
$$

where $q$ is the flux, $\Psi$ is the convective heat transfer coefficient, and $T_{s}$ the ambient temperature. The SPH formulation of Eq. 1 for a particle $i$ at time $n$ is expressed as

$$
\rho_{i} c_{i} \frac{T_{i}^{n+1}-T_{i}^{n}}{\Delta t}=\frac{\partial \kappa^{n}\left(T_{i}, \boldsymbol{x}_{i}\right)}{\partial \boldsymbol{x}_{i}} \sum_{j=1}^{N} \frac{m_{j}}{\rho_{j}} T_{j}^{n} \frac{\partial W_{i j}}{\partial x_{i j}}+\left(\kappa_{i}^{n}\left(T_{i}, \boldsymbol{x}_{i}\right)+\kappa_{j}^{n}\left(T_{j}, \boldsymbol{x}_{j}\right)\right) \sum_{j=1}^{N} \frac{m_{j}}{\rho_{j}} \frac{T_{i}^{n}-T_{j}^{n}}{x_{i j}} \frac{\partial W_{i j}}{\partial x_{i j}} .
$$

Here we show the equation for the explicit Eulerian method. In this equation, $m_{j}$ is the mass of particle $j, N$ is the number of particles, $W\left(x_{i j}, h\right)$ is a smoothing kernel function that is continuous in its influence domain with $h$ as the smoothing length, and $x_{i j}$ is the magnitude of the distance between particles $i$ and $j$. In this study, the following spline function is selected as the kernel function:

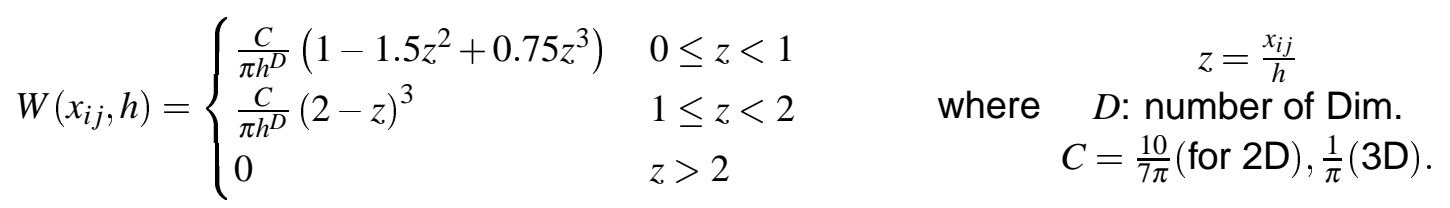

The first term on the right hand of Eq. 3 is for the case when the conductivity is a function of the coordinate. The second term is the second order differential term, which can also be expressed by the second derivative with respect to the kernel function. We use the present form to correspond to cases in which the thermal conductivity is a discrete type, as well as for steady problems.

The treatment of the Dirichlet boundary has no problem simply by the SPH; however, the accuracy is decreased if not treated with particular care for flux based boundary conditions. Here we select the CSPM formulation. The Taylor series expansion for a function $f$ up to the first derivative using the particle method is expressed as

$$
\sum_{j=1}^{N} \frac{m_{j}}{\rho_{j}} \Delta x_{\alpha} W_{i j, \beta} f_{i, \alpha}=\sum_{j=1}^{N} \frac{m_{j}}{\rho_{j}}\left(f_{i}-f_{j}\right) W_{i j, \beta}
$$

The flux based boundary conditions can then be written as

$$
-\kappa_{i} \frac{\partial T_{i}(\boldsymbol{x}, t)}{\partial n}=\sum_{k=1}^{D}\left(T_{i}-T_{j}\right) \Phi_{\alpha \beta} \boldsymbol{n}^{k}=B \cdot h
$$

where $\boldsymbol{x}$ is a point on the boundary, $\boldsymbol{n}^{k}$ is outward unit normal vector of the $k$ direction, $\Phi_{\alpha \beta}$ is a coefficient produced by solving $f_{i, \alpha}$ in Eq. 5, $B$ is a coefficient matrix with $D \times D(D$ is the number of dimension) terms. Furthermore, $h=q_{0}$ when the flux is designated and $h=\Psi\left(T_{s}-T_{i}(\boldsymbol{x}, t)\right)$ under convective conditions .

Equation 3 is for a non-steady problem and can be used to solve a steady problem by allowing the left hand term equal zero. The equation then becomes a set of linear simultaneous equations when the conductivity is only a function of the coordinates. For a temperature dependent case, the equation becomes a nonlinear problem, which is also solvable; however, the technique for this solution is beyond the scope of this paper.

\section{Model Calculation for Heat Transfer Problem}

Firstly we analyze problems in which the conductivity $\kappa$ is coordinate dependent, and investigate the relationship between the number of particles and the accuracy. The model is a $1 \times 1$ square domain. The adiabatic boundaries are up and low edges and the isothermal boundaries are $T=10$ at left and $T=0$ at right. In the all domain $\kappa=A e^{B x}$. The steady problem was solved with $A=1$ and $B=2,-2$, comparing to theoretical solution shown in Fig. 1 . This figure shows the case for $11 \times 11$ number of 


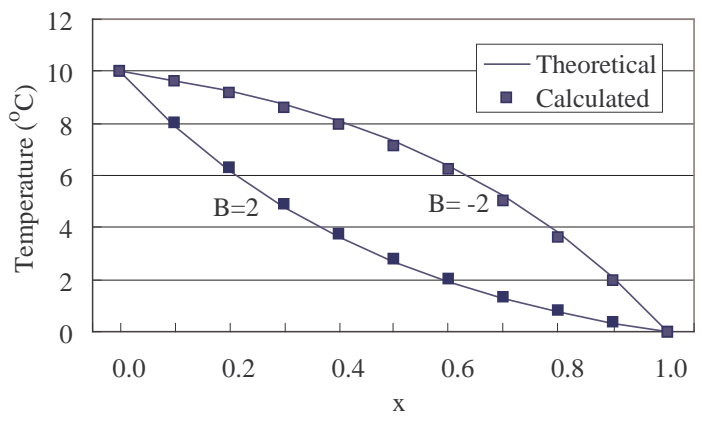

Figure 1: Distribution of $T, \kappa=e^{B x}$, solved as a steady problem

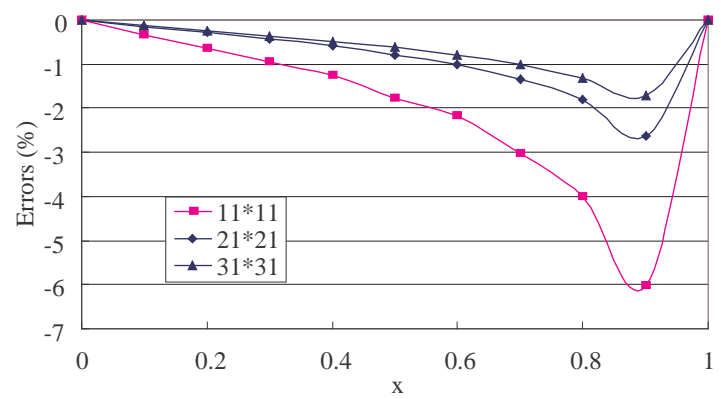

Figure 2: Error behavior according to particle number, $\kappa=e^{-2 x}$

particles. The accuracy will be improved if the number of particles is increased, as illustrated in Fig. 2. Because the analytical solution for the non-steady problem is not available, it was calculated up to the steady state; however, there is no significant difference between the two calculations.

The next calculation is for the case where the conductivity is temperature dependent, which was calculated up to the steady state and compared to the results for the steady problem, as for the previous example. Figure 3 displays calculated versus the analytical results when the domain is the same as the previous example, except that the left and right boundaries are $T=0$ and $T=1$, respectively, and $\kappa=e^{a T}(a=4)$ in the $x$ direction. The largest error occurs at the point of $x=0$, where the absolute value of $T$ is the smallest, as for the previous example, and the order is very close to that of the previous example.

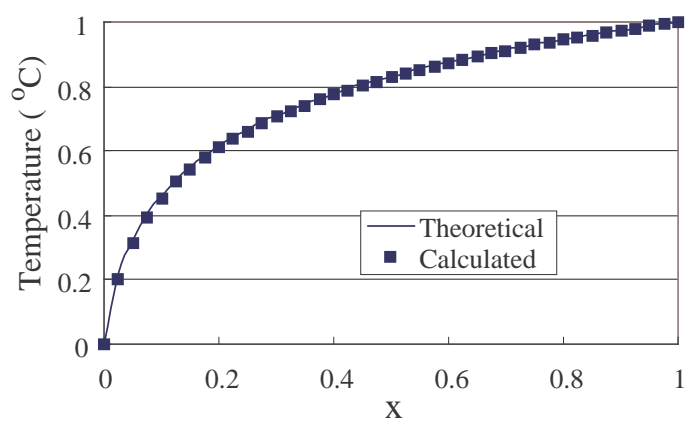

Figure 3: Distribution of $T, \kappa=e^{4 T}$

Equation 6 is tested using a model in which the flux $q=10 \mathrm{~W} / \mathrm{m}^{2}$ is applied to the cylindrical surface of a cylinder with radius $1 \mathrm{~m}$ and height $z=0.25 \mathrm{~m}$, where the initial temperature of all the domain is $T_{0}=0^{\circ} \mathrm{C}$. Parameters of $\rho=7860 \mathrm{~kg} / \mathrm{m}^{3}, \kappa=41.26 \mathrm{~W} / \mathrm{m} \mathrm{K}, c=0.1146 \mathrm{~J} / \mathrm{kg} \mathrm{K}$ are adopted. Figures 4 and 5 show the results from SPH and Eq. 6, respectively. The results indicate that the accuracy is significantly improved using CSPM.

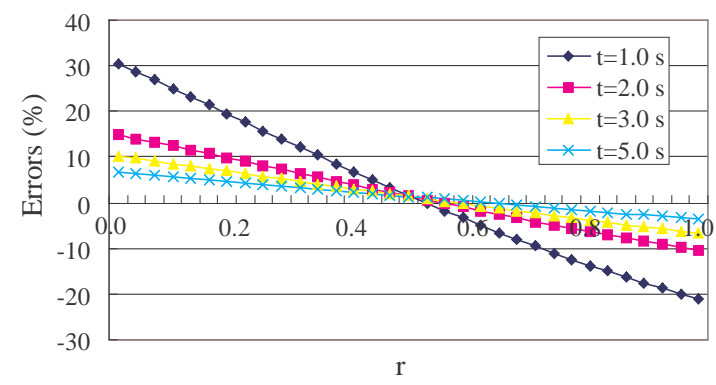

Figure 4: Distribution of $T$. The flux $q_{0}=10$ is given for a cylindrical surface using the SPH formulation

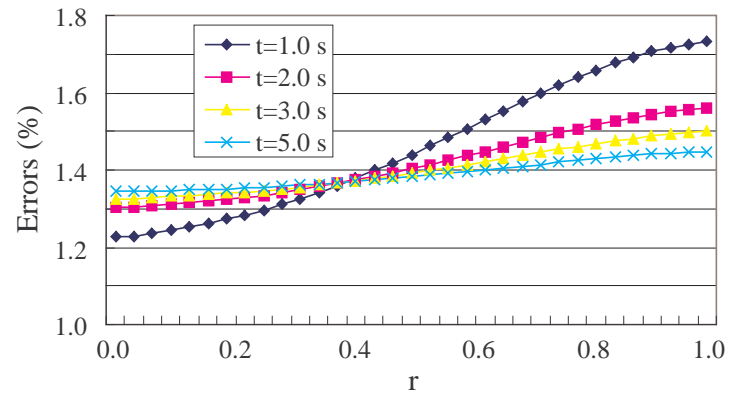

Figure 5: The same case as in Figure 4, but calculated using the SPH-CSPM formulation

\section{Calculation of the thermal shock problem}

The last calculation will be compared to an experiment reported by Sadowski et al [6]. The sample is a $30 \mathrm{~mm}$ diameter circular plate comprised of five ceramic layers (each $0.5 \mathrm{~mm}$ thick); a pure $\mathrm{Al}_{2} \mathrm{O}_{3}$ layer 
and composite layers consisting of $\mathrm{Al}_{2} \mathrm{O}_{3}$ and 5, 10, 15, $20 \mathrm{wt} \% \mathrm{ZrO}_{2}$. The plate is preheated to 1023, 1073 , and $1123 \mathrm{~K}$ respectively, then a high-velocity room temperature nitrogen jet is channeled onto the center of the upper disk surface for approximately $20 \mathrm{~s}$ using a metal tube (inner diameter $\mathrm{D}=4.5 \mathrm{~mm}$ ) placed perpendicular and $3 \mathrm{~mm}$ above the surface. The variation of the temperature during this sudden cooling process is recorded at the center of the plate (point 1), and at a point $14.5 \mathrm{~mm}$ from the center (point 2), on the opposite side of the plate. From the calculation of Sadowski et al, the variation of conductivity $\kappa$ in the direction of thickness $(z)$ is omitted; the $\kappa$ of $\mathrm{ZrO}_{2}$ is constant and that of $\mathrm{Al}_{2} \mathrm{O}_{3}$ is $\kappa^{A l_{2} O_{3}}(T)=5.85+26524.4 \exp (-0.002 T) /(242.85+T)$, and the all thermal properties including $\kappa$ were evaluated according to the linear rule of mixture. In regard to the boundary conditions, the cooled surface and the cylindrical surface are convective boundaries, with the convective coefficient varied in the radial direction, and the opposite surface is adiabatic. The model is independent of $\theta$, so that a 2-D $z-r$ system was used to simplify the calculation.

In our calculation, the variation of $\kappa$ in the $z$ direction is also considered and we set the $\kappa$ as

$$
\kappa(T, z)=\kappa^{\mathrm{Al}_{2} \mathrm{O}_{3}}(T)(1-k z)+\kappa^{\mathrm{ZrO}_{2}} \cdot k z,
$$

where $k$ is the rate of change of the component. Other parameters are the same as Ref. [7]. Rather than create a special code for a $z-r$ system, we produce the model from a number of circles of equally spaced particles, so that the radially changed heat convective condition can be used readily with a conventional calculation system. The diameter of the particles is $0.5 \mathrm{~mm}$, and the time step is $0.01 \mathrm{~s}$ which is recommended by Ref. [8]. The simplest explicit Eulerian method is used to show that even this method can provide reasonable accuracy.

Figure 6 presents the calculated and experimental temperature profiles during the cooling process at the two points, with $T_{0}=1023 \mathrm{~K}$, in which the difference from the experimental data is the largest in the three cases. It can be seen that the behavior of the two calculated curves is similar to the experimental ones, correspondingly, and the maximum difference of the calculated data is approximately $1.5 \%$ from the experimental data (point 2), which is better than that reported in either Refs. [4] or [7]. Graphs of other two cases are omitted due to the space limit. However, there is almost no difference if $\kappa$ is treated the same as in Ref. [7]. This is $K$

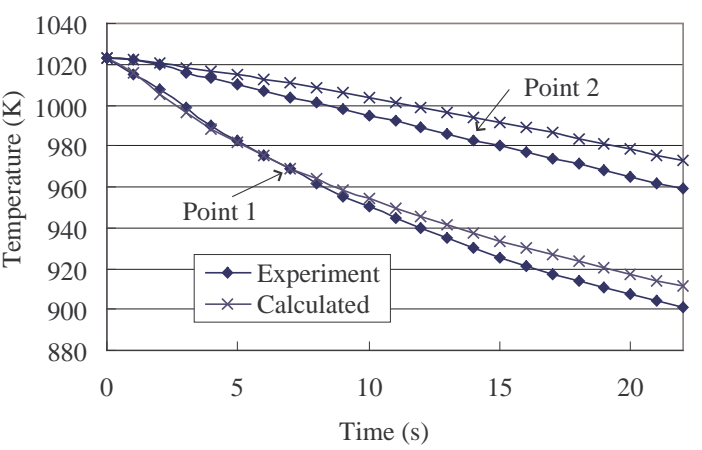

Figure 6: Calculated an experimental temperatures at points 1 and 2 for an initial temperature of $T_{0}=1023$ because the sample is sufficiently thin enough to be approximated to a homogeneous geometry. It can be concluded that the computing system utilized in this study is sufficient for calculation of the thermal performance of FGMs, and is expected to be available for more complex cases.

\section{Conclusions}

The accuracy was improved by the SPH-CSPM formulation and its practicality was verified for both steady and non-steady heat transfer problems.

\section{References}

[1] Y. Ochiai: Engineering Analysis with Boundary Elements 28 (2004) 1445-1453.

[2] H. Sakurai: WIT Trans. Eng. Sci., 64 (2009) 45-54.

[3] J. Sladek, V. Sladek, Ch. Zhang: Comput. Mater. Science 28 (2003) 494-504.

[4] K. Nakonieczny and T. Sadowski: Comput. Mater. Science 44 (2009) 1307-1311.

[5] Kamran, A. Khan, Anastasia H. Muliana: Composites Part B 40 (2009) 511-521.

[6] T. Sadowski, M. Boniecki, Z. Librant, K. Nakonieczny: Int. J. Heat Mass Transfer 50 (2007) 44614467.

[7] T. Sadowski, S. Ataya, K. Nakonieczny: Comput. Mater. Science 45 (2009) 624-632.

[8] P. W. Cleary and J. J. Monaghan: J. Comput. Phys. 148 (1999) 227-264. 